\title{
Operative Identifizierung linearer Modelle von technisch- ökonomischen Entwicklungssystemen bei plötzlicher Ände- rung der Koeffizienten der Produktionsmatrix
}

\author{
Dr. rer. techn. Michail Zygankow
}

\section{Modellierung linearer Systeme}

Eine breite Klasse technisch-ökonomischer und technologischer Systeme wird im stationären Betrieb durch lineare Modelle beschrieben. Diese durch Reduzierung genormten Modelle (durch Einfiihrung einer definierten Anzahl von zusätzlichen Variablen) können zweckmäßigerweise in der Matrizenschreibweise dargestellt werden:

$$
\mathrm{A} \cdot \mathrm{x}=\mathrm{b} \quad \mathrm{f}=\mathrm{c}^{\mathrm{T}} \cdot \mathrm{x} \quad \mathrm{x} \geq 0 \quad \mathrm{~b} \geq 0
$$

Die Aufgabe besteht darin, die realisierbaren Formen technisch-ökonomischer Aktivitäten (Produktionsverfahren) so zusammenstellen, daß ein Effektivitätsmaximum (max f) nach dem Rentabilitäts- oder dem Produktivitätskriterium $\mathrm{f}$ o. ä. gewährleistet ist.

Die Matrix A wird als technologische Matrix verstanden, der Vektor b ist der Kapazitätsvektor (im breiteren Sinne - Faktorenvektor), der x-Vektor ist die Spalte der Intensität der Inanspruchnahme der technologischen Produktionsverfahren und der Zeilenvektor $c^{\mathrm{T}}$ enthält die Deckungsbeiträge der Verfahren. Die Größe der Matrix $A(m \times n)$ kennzeichnet den Verbrauch $m$ verschiedener Arten von Ressourcen für $\mathrm{n}$ unterschiedliche Formen technologischer Aktivitäten (Produktionsverfahren). Die Koeffizienten $a_{i j}$ dieser Matrix werden als Verbrauchskoeffizienten für die i-te Ressourcenart, die für das Funktionieren des j-ten Produktionsverfahrens bei einfacher Intensität erforderlich ist. Sie charakterisieren den spezifischen Energieinhalt, die Materialintensität, die Arbeitsintensität sowie die Produktivität der Ausriistung. Sie bewerten also die Ressourcenintensität der Verfahren nach der einen oder der anderen Ressourcenart.

Für kontinuierliche chemisch-technologische und chemisch-ökonomische Prozesse werden die Varialblen $\mathrm{x}$ und die Koeffizienten c und b als festgelegte oder maximal zulässige Ressourcenverbrauchsgeschwindigkeit betrachtet. Als nahezu kontinuierlich können Prozesse der Massen- oder Großserienproduktion im Maschinenbau und anderen Industriezweigen betrachtet werden.

\section{Nichtstationäres Verhalten „technologi- scher" Eigenschaften von gesteuerten Systemen}

Die technologische Matrix bestimmt die „technologischen" Eigenschaften des Steuerobjekts, die unter Produktionsbedingungen nicht konstant bleiben. Aus welchen Gründen auch immer (physische Alterung der Ausrïstung durch den Teilverschleiß, instabile Rohstoffei- genschaften oder konjunkturelle Schwankungen) kommt es zu bestimmten Schwankungen der spezifischen Ressourcenverbrauchskoeffiziente. Dies führt dazu, daß sich die Koeffizienten $\mathrm{a}_{\mathrm{ij}}$ mit der Zeit ändern. Der Charakter der Koeffizientenvariierung kann unterschiedlicher Natur sein.

Die Zeitfunktionen $\mathrm{a}_{\mathrm{ij}}(\mathrm{t})$ können determinierte oder stochastische Prozesse darstellen, sie können glatt oder unterbrochen sein. Die stuickweise konstante Steuerung (Abb. 1) ist für Lösungen kurzer Zeitabstände innerhalb „längerer" Intervalle charakteristisch (z. B. für das Verhältnis zwischen operativer und strategischer Steuerung). Für eine Steuerung dieser Art ist es zweckmäßig, die Ressourcenverbrauchskoeffiziente $\mathrm{a}_{\mathrm{ij}}$ als stückweise konstante Funktionen darzustellen.

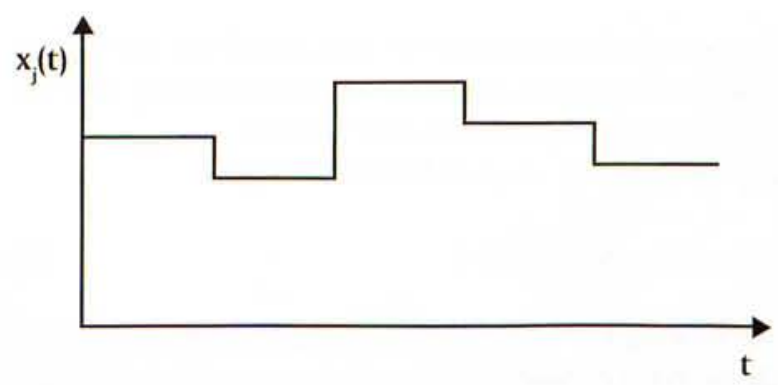

Abb. 1: Beispiel einer stückweise konstanten Steuerung

\section{Einfluß des nichtstationären Verhaltens auf die Stabilität der Steuerung}

Ziel der operativen Planung ist es, das Maximum des Effektivitätskriteriums $\mathrm{f}$ bei laufenden Werten von $\mathrm{a}_{\mathrm{ij}} \mathrm{zu}$ erreichen. Deshalb wird die optimale Steuerung $\mathrm{x}^{*}(\mathrm{t})$ und ihre Änderung in der Zeit durch ein Variieren dieser Größen bestimmt. Da das Modell (1) linear ist, wird die Steuerung in jedem Abschnitt mit konstanten Koeffizienten der Matrix A durch Umkehrung der optimalen Basismatrix $A_{b}$, die aus $A$ ermittelt wurde, determiniert. Allgemein kann angenommen werden, daß die optimale Basis sich aus der ersten n Spaltenvektoren der Matrix A zusammensetzt. Folglich kann das System der Nebenbedingungen $A \cdot x=b$ im Modell (1) folgendermaßen dargestellt werden kann:

$A_{b} \cdot x_{b}+A_{n} \cdot x_{n}=b$

wobei $A_{b}$ und $A_{n}$ - Blöcke der Matrix $A$ sind, die den Vektoren von Basis- und Nichtbasis-Variablen $x_{b}$ und $x_{n}$ entsprechen. 
Das heißt:

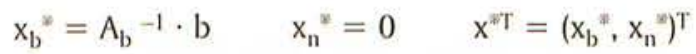

wobei * die optimale Lösung

und ${ }^{\mathrm{T}}$ das Transponieren symbolisieren.

Wenn der Trend der Änderung von $\mathrm{a}_{\mathrm{ij}}(\mathrm{t})$ der Rohstoffverbrauchsnormen zu Beginn der strategischen Planung bekannt ist, dann ist auch eine optimale Lösung $x^{*}(t)$ für diesen Abschnitt möglich. Wenn jedoch $\mathrm{a}_{\mathrm{ij}}$ von plötzlichen nicht vorhersehbaren Änderungen behaltet wird, dann ist nur für den bevorstehenden Zeitabschnitt eine Korrektur der bereits ausgearbeiteten Lösung möglich.

Es ist sinnvoll, die Korrektur auf der Grundlage bekannter Sensivitätsbedingungen der Basis und der Basislösungen vorzunehmen:

$c^{\mathrm{T}}{ }_{\mathrm{n}}-\left(\mathrm{c}_{\mathrm{b}}^{\mathrm{T}} \cdot \mathrm{A}_{\mathrm{b}}{ }^{-1} \cdot \mathrm{A}_{\mathrm{n}}\right) \leq 0 \quad \mathrm{x}_{\mathrm{b}}{ }^{*}=\mathrm{A}_{\mathrm{b}}{ }^{-1} \cdot \mathrm{b} \quad \mathrm{x}_{\mathrm{b}}{ }^{*} \geq 0$

wobei $\mathrm{c}_{\mathrm{b}}^{\mathrm{T}}$ der Zeilenvektor der Basiskomponenten des Vektors $\mathrm{c}$ ist.

$\mathrm{c}_{\mathrm{b}}^{\mathrm{T}} \cdot \mathrm{A}_{\mathrm{b}}{ }^{-1}=1$ ist der Zeilenvektor der optimalen Preise bzw. der Variablen der Aufgabe nach dem Modell (1) ähnlich ist.

Die erste Unlgeichung (4) ist ausgehend von den Sensivitätsbedingungen für eine optimale Lösung nur für Nicht-Basis-Komponenten des Vektors

$c_{n}^{T}-\left(c_{b}^{T} \cdot A_{b}-1 \cdot A_{n}\right)$ zu berüicksichtigen:

$c^{T}{ }_{n}-\left(c^{T}{ }_{b} \cdot A_{b} \cdot A_{n}\right)^{T} \leq 0$

Die Lösung $x^{* T}=\left(x_{b}, x_{n}\right)^{T}$ ändert sich nicht, wenn die Elemente der Matrix $A_{n}$ so variiert werden, daß die Ungleichung (5) güiltig bleibt. Das Variieren von Komponenten der Basis-Vektoren der Matrix $A_{b}$ führt zur Änderung der Basis-Lösung. Das folgt aus der Bedingung $x_{b}=A_{b}-1 \cdot b$ des Systems (4). Wenn die Ungleichung $x_{b} \geq 0$ gültig bleibt, kann die Basis unverändert bleiben.

In allen anderen Fällen ändern sich sowohl die Struktur der Basis-Vektoren als auch die konkrete Werte der verbleibenden „aiten“ Vektoren. Dies begründet die Wichtigkeit der laufenden Kontrolle der Ressourcenverbrauchskoeffitiente $\mathrm{a}_{\mathrm{ij}}$. In einigen Fällen (s. u.) kann jedoch eine solche Kontrolle mit Schwierigkeiten verbunden sein.

Es ist offensichtlich, daß auf der Grundlage der Kenntnis des möglichen Schwankungsbereichs von $\mathrm{a}_{\mathrm{ij}}$ diese Schwierigkeit durch folgende Maßnahmen gelöst werden kann:

- Festlegen von Grenzwerten für Koeffizienten der technologischen Matrix,

- Einführung zusätzlicher Variablen

- Umformung des Systems (1) derart, daß die Forderungen für ein garantiertes Ergebnis bei nichtstationären Bedingungen erfuillt sind.

Dieses Herangehen kann jedoch zu wesentlichen Verlusten für das Optimalkriterium f führen.

\section{Aufgabe der indirekten Kontrolle von Ressourcenverbrauchsnormen}

Ferner werden Bedingungen für plötzliche Änderungen von einem oder einigen Koeffizienten $\mathrm{a}_{\mathrm{ij}}$ behandelt. Diese Änderung kann nur durch ein Variieren von Komponenten des Vektors $A_{b} \cdot x_{b}=b, d$. h. einer limitierten Gesamtgeschwindigkeit des Ressourcenverbrauchs bestimmt werden. Dies ist für Systeme charakteristisch, aus denen die Strukturelemente, die „physisch“ den Elementen der Matrix A entsprechen, nicht klar hervorgehen. Eine direkte Messung von Ressourcenverbrauchskoeffizienten ist daher unmöglich.

Als praktisches Beispiel für ein derart gesteuertes Objekt kann man sich einen technologischen Apparat vorstellen, der Energie verbraucht und der eine Anzahl von Eingängen für den Materialfluß hat. Jeder einzelne Materialfluß wird durch $x_{j}$ beschrieben. Eine Änderung des Energieverbrauchs, die vom j-en technologischen Fluß verursacht wird, kann nur als Gesamtänderung des Energieverbrauchs fuir den Apparat registriert und subjektiv jedem der Eingänge zugeordnet werden.

Daraus ergibt sich die Aufgabe der Ermittlung der Matrix A nach den passiven Beobachtungen oder durch spezielle Prüfung. Die Ermittlung der Matrix A im System (1) hat eine Reihe von spezifischen Eigenschaften, die einerseits mit der Lösungsart der linearen Programmierung und andererseits mit dem Anwendungsverfahren für ein real gesteuertes Objekt verbunden sind.

\section{Besonderheiten des Systemtests (1) während der Steuerungsoptimierung}

Eine Änderung der Ressourcenverbrauchskoeffizienten während der Steuerungsoptimierung kann nur für grundlegende technologische Produktionsverfahren erfaßt werden. Sie erscheint entsprechend der Bedingung $\mathrm{x}_{\mathrm{b}}{ }^{*}=\mathrm{A}_{\mathrm{b}}{ }^{-1} \cdot \mathrm{b}$ des Systems (4) und kann in einer Vektor- oder Skalarform ausgedrüickt werden:

$A_{b} \cdot x_{b}{ }^{*}=b$

$\Sigma \mathrm{ab}_{\mathrm{ij}} \times \mathrm{x}_{\mathrm{bj}}{ }^{*}=\mathrm{b}_{\mathrm{i}}$

wobei die Addition über alle Indizes der Basis-Variablen erfolgt.

$\mathrm{a}_{\text {bij }}$ stellt die Komponenten der Basis-Vektoren der optimalen Basis der technologischen Matrix dar, $\mathrm{x}_{\mathrm{bj}}{ }$ " bezeichnet die optimalen Werte für Vektor-Komponenten der Basis-Variablen und $b_{i}$ Vektor-Komponenten für limitierte Werte der Ressourcenverbrauchsgeschwindigkeiten (Faktoren) in der Produktion.

Aufgrund $\mathrm{x}_{\text {nij. }}{ }^{*}=0$ erscheint keine Änderung von $\mathrm{a}_{\text {nij }}$. wenn keine Änderung von $x_{n j}$ durch einen Test vorgenommen wird.

Ist der Index k für ein einzelnes technologisches Produktionsverfahren bekannt, dessen Wert $\mathrm{ab}_{\mathrm{ik}} \mathrm{um} \Delta \mathrm{ab}_{\mathrm{ij}}$ verändert ist, so kann der Wert $\Delta \mathrm{ab}_{\mathrm{ij}}$ ohne Schwierigkeiten nach der Beendigung der Kontrolle von $b_{i}$ bestimmt werden: 
$\Delta \mathrm{a}_{\mathrm{bij}}=\Delta \mathrm{b}_{\mathrm{i}} / \mathrm{x}_{\mathrm{b}}{ }^{*} \mathrm{k}$

wobei $\Delta b_{i}$ die registrierte Änderung der Ressourcenverbrauchsgeschwindigkeit ist.

Wenn aber k unbekannt ist, so ist eine Prïung der Verfahren durch eine Änderung der Variablen xk um den Wert $\Delta \mathrm{x}_{\mathrm{k}}$ erforderlich. Das führt zu einer zusätzlichen Geschwindigkeitsänderung für den Ressourcenverbrauch:

$\Delta \mathrm{b}_{\mathrm{bik}}=\left(\mathrm{a}_{\mathrm{ik}}+\Delta \mathrm{a}_{\mathrm{ik}}\right) \cdot \Delta \mathrm{x}_{\mathrm{k}}$

Die Werte $\Delta \mathrm{a}_{\text {bik }}$ können aus folgender Gleichung berechnet werden:

$\Delta \mathrm{a}_{\mathrm{ik}}=\left(\Delta \mathrm{b}_{\mathrm{bik}}-\mathrm{a}_{\mathrm{ik}} \cdot \Delta \mathrm{x}_{\mathrm{k}}\right) / \Delta \mathrm{x}_{\mathrm{k}}$

Bei der Auswahl der Srittwerte $\Delta \mathrm{x}_{\mathrm{k}}$ ist es erforderlich, den Umstand zu berücksichtigen, daß $\Delta \mathrm{a}_{\mathrm{ik}}$ bei Auswahl eines zu kleinen absoluten Wertes von $\Delta x_{k}$, der vom Rauschen des gesteuerten Objekts ïberdeckt wird nicht genau ermittelt werden kann. Die Begründung liegt darin, daß $\Delta \mathrm{a}_{\mathrm{k}} \cdot \Delta \mathrm{x}_{\mathrm{k}}$ eine unendliche kleine Größe zweiter Ordnung im Verhältnis zum linearen Teil des Abweichung $\mathrm{a}_{\mathrm{ik}} \cdot \Delta \mathrm{x}_{\mathrm{k}}$ ist. Ein zu großer Wert von $\Delta \mathrm{x}_{\mathrm{k}}$ würde $z u$ einem wesentlichen Verlust bei der Berechnung des Optimalkriteriums führen.

Änderungen $\Delta \mathrm{a}_{\mathrm{ik}}$ für mehrere technologische Verfahren können die Zeit der Ermittlung stark verzögern, weil die Kontrolle der $b_{i}$-Werte erst nach dem Ablauf der Abrechnungsperiode möglich ist. In dieser Zeit kann es zu neuen Änderungen von Verbrauchskoeffizient $\mathrm{a}_{\mathrm{ik}}$ kommen.

Die Aufgabe wird noch komplizierter bei einer automatischen Optimierung der Steuerung durch eine Stabilisierung des Vektors b entsprechend der Gleichung $\mathrm{x}_{\mathrm{b}}{ }^{*}=\mathrm{A}_{\mathrm{b}}{ }^{-1} \cdot \mathrm{b}$. Im letzten Fall ist die Ermittlung der Matrix $A_{b}{ }^{-1}$ durch Generieren von unterschiedlichen n Varianten von b durch eine Einführung von kleinen $\ddot{n} n$ derungen $\Delta \mathrm{b}_{\mathrm{i}}$ in die Komponenten des Vektors möglich. Die daraus folgende Matrizengleichung $X=A_{b}{ }^{-1}$. B wird nach $A_{b}{ }^{-1}$ umgestellt:

$X=A_{b}^{-1} \cdot B$

$X \cdot B-1=A_{b}-1 \cdot B \cdot B-1$

$X \cdot B^{-1}=A_{b}-1$

In dieser Gleichung werden die Spalten der Matrix X durch die $\mathrm{x}_{\mathrm{bk}}$-Werte gebildet, die sich aus der Substitution von b- durch $b_{k}$-Vektoren ergeben. Die Matrix B kann immer als nichtsinguläre Matrix konstruiert werden, z. B. durch Einfügen von Spalten b in jede Spalte der Diagonalmatrix mit Diagonalelementen $\Delta b_{i i}=\Delta b_{i}$.

Die gemäß (6) korrigierte Matrix $A_{b}{ }^{-1}$ kann für die Berechnung des neuen Optimums $x_{b}{ }^{"}=A_{b}{ }^{-1} \cdot b$ verwendet werden.

Um das Optimum für den neuen Wert $\mathrm{x}_{\mathrm{b}}{ }{ }^{\mathrm{z}} \mathrm{zu}$ garantieren, ist es erforderlich, die Bedingungen für die Erhaltung der optimalen Basis zu prüfen. Die Bedingung $\mathrm{x}_{\mathrm{b}}{ }^{\circ} \geq 0$ kann problemlos mit Hilfe des berechneten Werts $\mathrm{x}_{\mathrm{b}}{ }^{*}$ geprüft werden. Die Bedingung (5)

$c^{T}{ }_{n}-\left(c^{T}{ }_{b} \cdot A_{b}{ }^{-1} \cdot A_{n}\right)^{T} \leq 0$ kann nur dann geprüft werden, wenn die Korrektur der Werte $A_{n}$ durchgefuihrt wurde, z. B. ausgehend von den Ergebnissen der Testeinwirkung von $\Delta \mathrm{x}_{\mathrm{k}}$ auf die Nicht-Basis-Variablen.

Der Effekt der Testwirkung von $\Delta \mathrm{x}_{\mathrm{k}}$ kann entweder anhand der verursachten Änderung $\Delta$ foder durch die Gleichung (5) nach der Präzisierung des Vektors $A_{n k}$ enstprechend der Gleichung $A_{b} \cdot \Delta x_{b}+A_{n} \cdot \Delta x_{n}=0$ geprüit werden:

$A_{\mathrm{nk}}=-\left(\mathrm{A}_{\mathrm{b}} \cdot \Delta \mathrm{x}_{\mathrm{b}}\right) / \Delta \mathrm{x}_{\mathrm{k}}$

Eine Einschränkung des Mittelwertes des Vektors B erweist sich als vorteilhaft für die Steuerung des Zufallsvektors B.

\section{Literaturverzeichnis}

Ju. M. Ermoljew u.a.: Matematitscheskije metody issledowanija operazij. Kiew: Wischtscha schkola. 1979, $312 \mathrm{~S}$.

I.M. Borzenko: Adaptazija, prognozirowanije i wybor reschenij w algoritmach uprawlenija technólógitscheskimi objektami. Moskva: Energoatomizdat, 1984, $144 \mathrm{~S}$.

\section{Verfasser}

Dr. rer. tech. Michail Zygankow Staatliche Technische Universität Jaroslawl Fachbereich für Technische Kybernetik Tel. 007 (0) 852 / 446623

\section{Übersetzung}

Dr. phil. Olga Rösch

Technische Fachhochschule Wildau

Sprachenzentrum

Tel. (03375) 508367 